\title{
Decoupling of $\mathrm{N}$-acetyl-aspartate and Glutamate Within the Dorsolateral Prefrontal Cortex in Schizophrenia
}

\author{
Jennifer M. Coughlin, M.D. ${ }^{1}$, Teppei Tanaka, M.D. ${ }^{1}$, Anouk Marsman, Ph.D. ${ }^{2}$, Hongxing \\ Wang, M.D., Ph.D. ${ }^{1}$, Susanne Bonekamp, Ph.D. ${ }^{2}$, Pearl K. Kim, B.A. ${ }^{1}$, Cecilia Higgs, M.H.S. \\ ${ }^{1}$, Mark Varvaris, B.A. ${ }^{1}$, Richard A.E. Edden, Ph.D. ${ }^{2}$, Martin Pomper, M.D., Ph.D. ${ }^{2}$, David \\ Schretlen, Ph.D. ${ }^{1}$, Peter B. Barker, D.Phil. ${ }^{2}$, and Akira Sawa, M.D., Ph.D. ${ }^{1}$ \\ ${ }^{1}$ Department of Psychiatry and Behavioral Sciences, Johns Hopkins Medical Institutions, \\ Baltimore, MD, USA \\ ${ }^{2}$ Russell H. Morgan Department of Radiology and Radiological Science, Johns Hopkins Medical \\ Institutions, Baltimore, MD, USA \\ ${ }^{3}$ Department of Health Sciences Informatics, Johns Hopkins Medical Institutions, Baltimore, MD, \\ USA \\ ${ }^{4}$ Department of Biostatistics, Johns Hopkins Medical Institutions, Baltimore, MD, USA
}

\section{Abstract}

\begin{abstract}
Aberrant function of glutamatergic pathways is likely to underlie the pathology of schizophrenia. Evidence of oxidative stress in the disease pathology has also been reported. N-Acetylaspartate (NAA) is metabolically linked to both cascades and may be a key marker in exploring the interconnection of glutamatergic pathways and oxidative stress. Several studies have reported positive correlation between the levels of NAA and Glx (the sum of glutamate and glutamine) in several brain regions in healthy subjects, by using proton magnetic resonance spectroscopy $\left(\left[{ }^{1} \mathrm{H}\right] \mathrm{MRS}\right)$. Interestingly, one research group recently reported decoupling of the relationship between NAA and Glx in the hippocampus of patients with schizophrenia. Here we report levels of NAA and Glx measured using $\left[{ }^{1} \mathrm{H}\right]$ MRS, relative to the level of creatine $(\mathrm{Cr})$ as an internal control. The dorsolateral prefrontal cortex (DLPFC) and anterior cingulate cortex (ACC) in 25 patients with schizophrenia and 17 matched healthy controls were studied. In DLPFC, NAA/Cr and $\mathrm{Glx} / \mathrm{Cr}$ were significantly positively correlated in healthy controls after correction for the effect of age and smoking status and after correction for multiple comparisons ( $\mathrm{r}=0.63, P=0.017)$. However, in patients with schizophrenia, the positive correlation between NAA/Cr and $\mathrm{Glx} / \mathrm{Cr}$ was not observed even after correcting for these two variables $(\mathrm{r}=-0.33, P=0.124)$. Positive correlation between NAA/Cr and Glx/Cr was not observed in the ACC in both groups. Decoupling of NAA and Glx in the DLPFC may reflect the interconnection of glutamatergic pathways and oxidative stress in the pathology of schizophrenia, and may possibly be a biomarker of the disease.
\end{abstract}

\footnotetext{
*Corresponding Author: Akira Sawa, M.D., Ph.D., 600 N. Wolfe Street, Meyer 3-166A, Baltimore, MD 21287, Tel: 410-955-4726, Fax: 410-614-1792, asawa1 @jhmi.edu.

Conflict of Interest

None
} 


\section{Keywords}

anterior cingulate cortex (ACC); dorsolateral prefrontal cortex (DLPFC); glutamate; N-acetylaspartate (NAA); oxidative stress; mitochondria; proton magnetic resonance spectroscopy ([ $\left.\left.{ }^{1} \mathrm{H}\right] \mathrm{MRS}\right)$; and schizophrenia (SZ)

\section{Introduction}

Alterations in glutamatergic pathways, including dysfunctional activity of N-methyl-daspartate (NMDA)-type glutamate receptors (NMDA-R), is likely to underlie the development and clinical course of schizophrenia. The effect of NMDA-R hypofunction on Y-aminobutyric acid (GABA) neurons may directly contribute to excitation/inhibition imbalance of the cerebral cortex, particularly in dorsolateral prefrontal cortex (DLPFC), and ultimately to cognitive deficits such as those of executive and working memory [1-4]. In parallel, involvement of oxidative stress in the pathophysiology of schizophrenia has also been proposed [5-11]. Oxidative stress may be increased by and further amplify functional deficits of mitochondria $[12,13]$. Indeed, some autopsy studies have reported mitochondrial deficits in the brains of patients with schizophrenia [14, 15].

Magnetic spectroscopy measures brain chemicals in regions of interest and may be a useful method to examine changes in key substances related to glutamatergic pathways and oxidative stress in schizophrenia. Nonetheless, difficulty in differentiating certain chemical peaks, such as those of glutamate and glutamine using proton magnetic resonance spectroscopy ( $\left.\left[{ }^{1} \mathrm{H}\right] \mathrm{MRS}\right)$ at low field strength remains a technical challenge. To date, studies of glutamate levels, quantified as the combination of measured glutamate and glutamine (Glx), in DLPFC reveal conflicting results [16]. Some studies show no change in Glx between patient and control groups, whereas some studies report increases or decreases in Glx in medicated subjects [17].

$\mathrm{N}$-acetylaspartate (NAA) is the second most abundant amino acid in human brain and can also be measured using $\left[{ }^{1} \mathrm{H}\right] \mathrm{MRS}$. The level of NAA is high in neurons and is sensitive to the effects of mitochondrial function and oxidative stress[18]. NAA and glutamate are tightly connected metabolically, in part through tricarboxylic acid (TCA) and glutamateglutamine cycles [19]. Accordingly, a significantly positive correlation between NAA/Cr (the level of NAA normalized by the level of creatine) and $\mathrm{Glx} / \mathrm{Cr}$ in the dorsal anterior cingulate cortex (ACC), pregenual ACC, cerebellum, and hippocampus has been demonstrated [20-23]. Thus, NAA might be a key molecule to explore the interconnected role of glutamatergic pathways and oxidative stress in schizophrenia. Of interest, a study has reported that such homeostatic relationship between NAA/Cr and $\mathrm{Glx} / \mathrm{Cr}$ is disrupted in the hippocampus of patients with schizophrenia[20].

Building on this background, the present study used $\left[{ }^{1} \mathrm{H}\right] \mathrm{MRS}$ to measure NAA/Cr and Glx/Cr in two cortical regions, DLPFC and ACC, in patients with schizophrenia and in healthy controls. Absolute concentrations and the correlation between NAA/Cr and $\mathrm{Glx} / \mathrm{Cr}$ were assessed in both cohorts. 


\section{Materials and Methods}

\section{Participants}

This study was approved by the Johns Hopkins Medicine Institutional Review Board. All participants provided written informed consent. Adult patients with schizophrenia were recruited from outpatient clinics, inpatient services, and psychiatric day hospitals affiliated with the Johns Hopkins Medical Institution and from hospitals in the surrounding greater Baltimore-Washington, D.C. area. Inclusion criteria included diagnosis of schizophrenia according to criteria of the Diagnostic and Statistical Manual of Mental Disorders-Fourth Edition (DSM-IV) after completion of diagnostic and clinical assessment administered by a research team psychiatrist (JC). This assessment included the Structured Clinical Interview for DSM-IV Axis I Disorders-Clinician Version (SCID-CV) [24], and Scales for the Assessment of Positive and Negative Symptoms (SAPS and SANS) [25]. Additional clinical details related to adherence with prescribed medications and smoking status was also collected in this interview. Patients were excluded if they had 1) history of neurological disorder, structural brain abnormality or history of traumatic brain injury with loss of consciousness; 2) history of special education or known learning disability; 3) history of inflammatory medical condition including but not limited to diabetes, HIV and/or hepatitis; 4) benzodiazepine use in the past six months; 5) substance abuse in the previous six months or any history of substance dependence; 6) metal in the body or any other contraindication to participation in magnetic resonance imaging (MRI); and 7) active pregnancy. Healthy adults were recruited from flyers posted in the greater Baltimore-Washington, D.C. area and by word of mouth. Healthy controls were excluded if they had any of the above-mentioned exclusion criteria or if they had any DSM-IV axis I disorder.

\section{Neuropsychological Assessment}

All participants completed a two-hour battery of neuropsychological tests to assess cognitive function in six domains, namely processing speed, attention/working memory, ideational fluency, executive function, verbal memory and visual memory, as previously described[26]. A list of the specific tests included in this battery are provided in Supplemental Table and were administered and scored according to standard instructions by the same study team neuropsychologist who was blind to the clinical and imaging data of all participants (MV). Factor scores were calculated for each domain after controlling for age, sex, race and premorbid intelligence based on a normative sample [27, 28]. Premorbid intelligence was estimated using the Hopkins Adult Reading Test [29].

\section{MR acquisition and analysis}

All investigations were performed on a 3T MR scanner (Philips Healthcare, Best, Netherlands) with a whole body transmit coil in combination with a 16-channel receive coil (Nova Medical Inc., Burlington, MA, USA). A $\mathrm{T}_{1}$-weighted magnetization-prepared rapid gradient-echo (MP-RAGE) sequence $(1 \mathrm{~mm}$ isotropic voxels, $\mathrm{TR}=8 \mathrm{~ms}, \mathrm{TE}=3.8 \mathrm{~ms}$, flip angle $=8^{\circ}, 256 \times 256$ acquisition matrix, FOV $256 \times 180 \times 150 \mathrm{~mm}^{3}$, SENSE factor 2) was obtained for anatomical reference. Spectroscopic voxels were positioned in the medial anterior cingulate cortex (ACC; $35 \times 35 \times 35 \mathrm{~mm}^{3}$ ) and in the right dorsolateral prefrontal cortex (DLPFC; $40 \times 35 \times 30 \mathrm{~mm}^{3}$ ). Spectra were acquired using a point-resolved 
spectroscopy sequence (PRESS; TR $=5000 \mathrm{~ms}, \mathrm{TE}=35 \mathrm{~ms}, 16$ averages with water suppression and 1 average without water suppression). Water suppression was achieved using variable pulse powers and optimized relaxation delays (VAPOR) presaturation pulses.

Spectra were analyzed using LCModel version 6.3 [30]. The basis set was created from simulated spectra from individual metabolites and included alanine, aspartate, creatine $(\mathrm{Cr})$, GABA, glucose, glutamine (Gln), glutamate (Glu), glycerophosphorylcholine (GPC), lactate, myo-inositol (mI), scyllo-inositol, NAA, N-acetyl-aspartyl-glutamate (NAAG), phosphorylcholine $(\mathrm{PCh})$, and taurine. Spectra were normalized to the unsuppressed water signal, yielding quantification of the total NAA signal (NAA+NAAG), creatine $(\mathrm{Cr})$, choline-containing compounds (Cho; PCh+GPC), Glx (Glu+Gln) and myo-inositol (mI) in institutional units. Note, the peak of NAAG is much smaller than that of NAA in these brain regions. Metabolite concentration uncertainties that exceeded a Cramèr-Rao lower bound (CRLB), as provided by LCModel, of $20 \%$ or more were considered poor quality and excluded from further analyses.

\section{Statistical Analysis}

Statistical analyses were performed using STATA 13.1 for Macintosh (STATA, College Station, Texas, USA). Group comparisons of demographic, clinical, and neurocognitive variables were calculated using independent $t$ tests for continuous variables, with Bonferroni-corrected significance in the comparison of neurocognitive domain scores set to $\mathrm{p}=0.008=(0.05 / 6)$. Fisher's exact test was used for comparisons of categorical data. Normality of the data was confirmed by plotting the Q-Q plot. We employed a multiple linear regression model to examine the difference in metabolite levels (NAA and Glx) in each region (ACC and DLPFC) in patients and controls, controlling for age and smoking status (current smoker and current non-smoker). The correlation of NAA and Glx in both control and schizophrenia cohorts were examined by partial correlations, controlling for age and smoking status, in each respective region with Bonferroni-corrected significance set to $P=0.025(=0.05 / 2)$.

\section{Results}

\section{Study population}

Seventeen healthy controls and twenty-five patients with schizophrenia were enrolled in this study. Two patients reported that they were not taking antipsychotic medications though both had been prescribed an atypical antipsychotic medication in the past year. The other 23 patients reported adherence with prescribed antipsychotic medications: 15 patients were taking one atypical antipsychotic medication, two patients were taking one first generation, typical antipsychotic, four patients were taking a regimen of one typical and one atypical antipsychotic, and two patients were taking two atypical antipsychotics. Additionally, since parallel use of antidepressant and mood stabilizing medications was permitted, four of these patients were also taking a serotonin reuptake inhibitor and one was treated with valproic acid. One healthy control subject declined to participate in MRS imaging of the DLPFC. Three patients with schizophrenia and three healthy control subjects refused neuropsychiatric assessment. One patient with schizophrenia had a peripheral nerve injury 
limiting his fine motor dexterity and therefore his performance in the domain of processing speed was excluded due to poor performance in subtests where fine motor skills were required.

Patients with schizophrenia and healthy control participants did not differ in age, gender, ethnicity, or years of education. A trend of diminished processing speed and ideational fluency was observed in the cohort of patients with schizophrenia compared to healthy controls, although this finding was not significant after correction for multiple comparisons (Table 1).

\section{Metabolites levels}

The spectra in each brain region (DLPFC and ACC) are depicted in Figures 1 and 2. NAA/Cr and Glx/Cr in DLPFC and ACC did not differ between healthy controls and patients with schizophrenia, even after controlling for age and smoking status (Table 2). In DLPFC, NAA/Cr and Glx/Cr were significantly positively correlated in healthy controls after correction for the effect of age and smoking status and after correction for multiple comparisons $(\mathrm{r}=0.63, P=0.017)$. In contrast, in patients with schizophrenia, the positive correlation between NAA/Cr and Glx/Cr was not observed even after correcting for these two variables ( $\mathrm{r}=-0.33, P=0.124)$ (Figure 3$)$. In the ACC, we failed to observe correlation between NAA/Cr and Glx/Cr in both groups when data were corrected for the effect of age and smoking status (HC: $\mathrm{r}=-0.00, P=0.996 ; \mathrm{SZ}: \mathrm{r}=-0.15, P=0.483)$.

\section{Discussion}

Here we report the disruption of the positive correlation between NAA/Cr and $\mathrm{Glx} / \mathrm{Cr}$ in the DLPFC in patients with schizophrenia, whereas the correlation is well maintained in the same brain region of healthy controls. The coupling of NAA/Cr and $\mathrm{Glx} / \mathrm{Cr}$ has been reported in several brain regions in healthy controls [20-23], and the present report confirms this positive correlation in the DLPFC of healthy individuals. In contrast to healthy controls, patients with schizophrenia have reportedly shown decoupling of the relationship between $\mathrm{NAA} / \mathrm{Cr}$ and $\mathrm{Glx} / \mathrm{Cr}$ in the hippocampus of medicated and unmedicated patients with schizophrenia [20,21]. Thus, our observation of the decoupling of these metabolites in DLPFC in schizophrenia consistently extends the pathological observation in a different brain area. These results may aid in building a working hypothesis wherein functional decoupling of NAA and Glx may occur across the brain, or at least the frontal cortex, of patients with schizophrenia.

Although such hypothesis is tempting, we need to pay attention to possible discrepancies in the published data in greater detail. In regard to possible correlation of NAA/Cr and $\mathrm{Glx} / \mathrm{Cr}$ in healthy subjects, although multiple studies have supported this observation, we failed to observe this in ACC, just as another group did in the medial prefrontal cortex and DLPFC [31]. If we compare the discrepancy between results of the study by Kraguliac et al. [20] who found a trend-level positive correlation in ACC and our results, there are two major differences in the study populations and voxel placement. First, our study was composed mostly of African American participants, whereas the healthy control participants in the 
other study were mostly Caucasian [20]. It would be important to pay attention to the study populations, given published reports of known genetic variants involving glutamatergic and metabolic cascades associated with schizophrenia [32-34]. Second, we chose to use larger voxel size $\left(3.5 \times 3.5 \times 3.5 \mathrm{~cm}^{3}\right)$ and more rostral placement of the ACC voxel, whereas the other study used a voxel size of $2.7 \times 2 \times 1 \mathrm{~cm}^{3}$ over the ACC. Anatomic and functional heterogeneity across the ACC is reflected in heterogeneity of neurometabolites across this structure as recently reported in a study at 7T [35]. Taken together, in order to pursue the hypothesis of disrupted coupling of NAA/Cr and Glx/Cr in schizophrenia, careful comparison and normalization of several variables, including ethnic background and voxel placement will be needed. Furthermore, many technological advances, including stronger magnetic field strength to differentiate glutamatergic peaks, will benefit future study.

While we implemented statistical methods to control for the independent effects of age and smoking status on correlation of NAA/Cr and $\mathrm{Glx} / \mathrm{Cr}$, we acknowledge that other confounding variables may affect these results. For example, all but two of the patients with schizophrenia were taking antipsychotic medications at time of participation, and all of the patients had taken antipsychotic medications in the past year. Short and long-term effects of antipsychotic medication treatment on regional NAA/Cr and $\mathrm{Glx} / \mathrm{Cr}$ in humans have been investigated but still unclear [36-38]. One study of six-month treatment with haloperidol or clozapine in rats did not alter striatal NAA/Cr and Glx/Cr when compared to untreated rats using in vivo $\left[{ }^{1} \mathrm{H}\right] \mathrm{MRS}$ [39]. Further longitudinal study of changes in regional metabolite levels in the human brain over the course of antipsychotic treatment may elucidate these effects in the future.

The role of NAA, despite the abundance in the brain, remains elusive. This amino acid is associated with mitochondrial function and oxidative stress [18]. NAA is linked to glutamate through several metabolic cascades, possibly functioning as a reservoir of glutamate [19, 40]. This functional role is analogous to that of glutathione (GSH), a key antioxidant in the brain, which is also metabolically associated with glutamate through a series of biochemical reactions [41]. Importantly, deficits of GSH are well documented in the pathophysiology of schizophrenia $[5,8]$. Complementary study of alterations in NAA and GSH in conjunction with that of glutamatergic signaling and oxidative stress might be a promising strategy to further elucidate disease mechanisms and biomarkers.

\section{Supplementary Material}

Refer to Web version on PubMed Central for supplementary material.

\section{Acknowledgments}

This work was supported by the National Institute of Mental Health (MH-084018, MH-094268 Silvo O. Conte center, MH-069853, MH-085226,MH-088753, MH-092443), as well as grants from Stanley (AS), S-R (AS), RUSK (AS), NARSAD (JC and AS), MSCRF (AS). 


\section{References}

1. Gandal MJ, Sisti J, Klook K, et al. GABAB-mediated rescue of altered excitatory-inhibitory balance, gamma synchrony and behavioral deficits following constitutive NMDAR-hypofunction. Transl Psychiatry. 2012; 2:e142. [PubMed: 22806213]

2. Murray JD, Anticevic A, Gancsos M, et al. Linking microcircuit dysfunction to cognitive impairment: effects of disinhibition associated with schizophrenia in a cortical working memory model. Cereb Cortex. 2014; 24(4):859-72. [PubMed: 23203979]

3. Uhlhaas PJ. Dysconnectivity, large-scale networks and neuronal dynamics in schizophrenia. Curr Opin Neurobiol. 2013; 23(2):283-90. [PubMed: 23228430]

4. Yizhar O, Fenno LE, Prigge M, et al. Neocortical excitation/inhibition balance in information processing and social dysfunction. Nature. 2011; 477(7363):171-8. [PubMed: 21796121]

5. Do KQ, Trabesinger AH, Kirsten-Kruger M, et al. Schizophrenia: glutathione deficit in cerebrospinal fluid and prefrontal cortex in vivo. Eur J Neurosci. 2000; 12(10):3721-8. [PubMed: 11029642]

6. Gawryluk JW, Wang JF, Andreazza AC, Shao L, Young LT. Decreased levels of glutathione, the major brain antioxidant, in post-mortem prefrontal cortex from patients with psychiatric disorders. Int J Neuropsychopharmacol. 2011; 14(1):123-30. [PubMed: 20633320]

7. Yao JK, Leonard S, Reddy R. Altered glutathione redox state in schizophrenia. Dis Markers. 2006; 22(1-2):83-93. [PubMed: 16410648]

8. Emiliani FE, Sedlak TW, Sawa A. Oxidative stress and schizophrenia: recent breakthroughs from an old story. Curr Opin Psychiatry. 2014; 27(3):185-90. [PubMed: 24613987]

9. Coughlin JM, Ishizuka K, Kano SI, et al. Marked reduction of soluble superoxide dismutase-1 (SOD1) in cerebrospinal fluid of patients with recent-onset schizophrenia. Mol Psychiatry. 2013; 18(1):10-1. [PubMed: 22349781]

10. Johnson AW, Jaaro-Peled H, Shahani N, et al. Cognitive and motivational deficits together with prefrontal oxidative stress in a mouse model for neuropsychiatric illness. Proc Natl Acad Sci U S A. 2013; 110(30):12462-7. [PubMed: 23840059]

11. Kano S, Colantuoni C, Han F, et al. Genome-wide profiling of multiple histone methylations in olfactory cells: further implications for cellular susceptibility to oxidative stress in schizophrenia. Mol Psychiatry. 2013; 18(7):740-2. [PubMed: 22925834]

12. Andreazza AC, Wang JF, Salmasi F, Shao L, Young LT. Specific subcellular changes in oxidative stress in prefrontal cortex from patients with bipolar disorder. J Neurochem. 2013; 127(4):552-61. [PubMed: 23692477]

13. Prabakaran S, Swatton JE, Ryan MM, et al. Mitochondrial dysfunction in schizophrenia: evidence for compromised brain metabolism and oxidative stress. Mol Psychiatry. 2004; 9(7):684-97. 643. [PubMed: 15098003]

14. Gigante AD, Andreazza AC, Lafer B, Yatham LN, Beasley CL, Young LT. Decreased mRNA expression of uncoupling protein 2, a mitochondrial proton transporter, in post-mortem prefrontal cortex from patients with bipolar disorder and schizophrenia. Neurosci Lett. 2011; 505(1):47-51. [PubMed: 22001364]

15. Maurer I, Zierz S, Moller H. Evidence for a mitochondrial oxidative phosphorylation defect in brains from patients with schizophrenia. Schizophr Res. 2001; 48(1):125-36. [PubMed: 11278159]

16. Marsman A, van den Heuvel MP, Klomp DW, Kahn RS, Luijten PR, Hulshoff Pol HE. Glutamate in schizophrenia: a focused review and meta-analysis of (1)H-MRS studies. Schizophr Bull. 2013; 39(1):120-9. [PubMed: 21746807]

17. Poels EM, Kegeles LS, Kantrowitz JT, et al. Imaging glutamate in schizophrenia: review of findings and implications for drug discovery. Mol Psychiatry. 2014; 19(1):20-9. [PubMed: 24166406]

18. Marenco S, Bertolino A, Weinberger DR. In vivo NMR measures of NAA and the neurobiology of schizophrenia. Adv Exp Med Biol. 2006; 576:227-40. discussion 361-3. [PubMed: 16802716] 
19. Moffett JR, Ross B, Arun P, Madhavarao CN, Namboodiri AM. N-Acetylaspartate in the CNS: from neurodiagnostics to neurobiology. Prog Neurobiol. 2007; 81(2):89-131. [PubMed: 17275978]

20. Kraguljac NV, Reid MA, White DM, den Hollander J, Lahti AC. Regional decoupling of N-acetylaspartate and glutamate in schizophrenia. Neuropsychopharmacology. 2012; 37(12):2635-42. [PubMed: 22805603]

21. Kraguljac NV, White DM, Reid MA, Lahti AC. Increased hippocampal glutamate and volumetric deficits in unmedicated patients with schizophrenia. JAMA Psychiatry. 2013; 70(12):1294-302. [PubMed: 24108440]

22. Waddell KW, Zanjanipour P, Pradhan S, et al. Anterior cingulate and cerebellar GABA and Glu correlations measured by (1)H J-difference spectroscopy. Magn Reson Imaging. 2011; 29(1):19_ 24. [PubMed: 20884148]

23. Walter M, Henning A, Grimm S, et al. The relationship between aberrant neuronal activation in the pregenual anterior cingulate, altered glutamatergic metabolism, and anhedonia in major depression. Arch Gen Psychiatry. 2009; 66(5):478-86. [PubMed: 19414707]

24. First, MBSR.; Gibbon, M.; Williams, JB. Structured Clinical Interview for DSM-IV Axis I Disorders. New York: Biometrics Research Department, New Yok State Psychiatric Institute; 2002.

25. Andreasen NC, Olsen S. Negative v positive schizophrenia. Definition and validation. Arch Gen Psychiatry. 1982; 39(7):789-94. [PubMed: 7165478]

26. Ojeda N, Pena J, Schretlen DJ, et al. Hierarchical structure of the cognitive processes in schizophrenia: the fundamental role of processing speed. Schizophr Res. 2012; 135(1-3):72-8. [PubMed: 22226902]

27. Parmenter BA, Testa SM, Schretlen DJ, Weinstock-Guttman B, Benedict RH. The utility of regression-based norms in interpreting the minimal assessment of cognitive function in multiple sclerosis (MACFIMS). J Int Neuropsychol Soc. 2010; 16(1):6-16. [PubMed: 19796441]

28. Testa SM, Winicki JM, Pearlson GD, Gordon B, Schretlen DJ. Accounting for estimated IQ in neuropsychological test performance with regression-based techniques. J Int Neuropsychol Soc. 2009; 15(6):1012-22. [PubMed: 19796440]

29. Schretlen DJ, Winicki JM, Meyer SM, Testa SM, Pearlson GD, Gordon B. Development, psychometric properties, and validity of the hopkins adult reading test (HART). Clin Neuropsychol. 2009; 23(6):926-43. [PubMed: 19191072]

30. Provencher SW. Estimation of metabolite concentrations from localized in vivo proton NMR spectra. Magn Reson Med. 1993; 30(6):672-9. [PubMed: 8139448]

31. Kegeles LS, Mao X, Stanford AD, et al. Elevated prefrontal cortex gamma-aminobutyric acid and glutamate-glutamine levels in schizophrenia measured in vivo with proton magnetic resonance spectroscopy. Arch Gen Psychiatry. 2012; 69(5):449-59. [PubMed: 22213769]

32. Fromer M, Pocklington AJ, Kavanagh DH, et al. De novo mutations in schizophrenia implicate synaptic networks. Nature. 2014; 506(7487):179-84. [PubMed: 24463507]

33. Singh S, Kumar A, Agarwal S, Phadke SR, Jaiswal Y. Genetic insight of schizophrenia: past and future perspectives. Gene. 2014; 535(2):97-100. [PubMed: 24140491]

34. Modinos G, Iyegbe C, Prata D, et al. Molecular genetic gene-environment studies using candidate genes in schizophrenia: a systematic review. Schizophr Res. 2013; 150(2-3):356-65. [PubMed: 24094883]

35. Dou W, Palomero-Gallagher N, van Tol MJ, et al. Systematic regional variations of GABA, glutamine, and glutamate concentrations follow receptor fingerprints of human cingulate cortex. J Neurosci. 2013; 33(31):12698-704. [PubMed: 23904606]

36. Bertolino A, Callicott JH, Mattay VS, et al. The effect of treatment with antipsychotic drugs on brain N-acetylaspartate measures in patients with schizophrenia. Biol Psychiatry. 2001; 49(1):39_ 46. [PubMed: 11163778]

37. Szulc A, Galinska B, Tarasow E, et al. The effect of risperidone on metabolite measures in the frontal lobe, temporal lobe, and thalamus in schizophrenic patients. A proton magnetic resonance spectroscopy (1H MRS). Pharmacopsychiatry. 2005; 38(5):214-9. [PubMed: 16189748] 
38. Ende G, Braus DF, Walter S, et al. Effects of age, medication, and illness duration on the $\mathrm{N}$-acetyl aspartate signal of the anterior cingulate region in schizophrenia. Schizophr Res. 2000; 41(3):38995. [PubMed: 10728716]

39. Lindquist DM, Dunn RS, Cecil KM. Long term antipsychotic treatment does not alter metabolite concentrations in rat striatum: an in vivo magnetic resonance spectroscopy study. Schizophr Res. 2011; 128(1-3):83-90. [PubMed: 21429713]

40. Clark JF, Doepke A, Filosa JA, et al. N-acetylaspartate as a reservoir for glutamate. Med Hypotheses. 2006; 67(3):506-12. [PubMed: 16730130]

41. Koga M, Serritella AV, Messmer MM, et al. Glutathione is a physiologic reservoir of neuronal glutamate. Biochem Biophys Res Commun. 2011; 409(4):596-602. [PubMed: 21539809] 
A

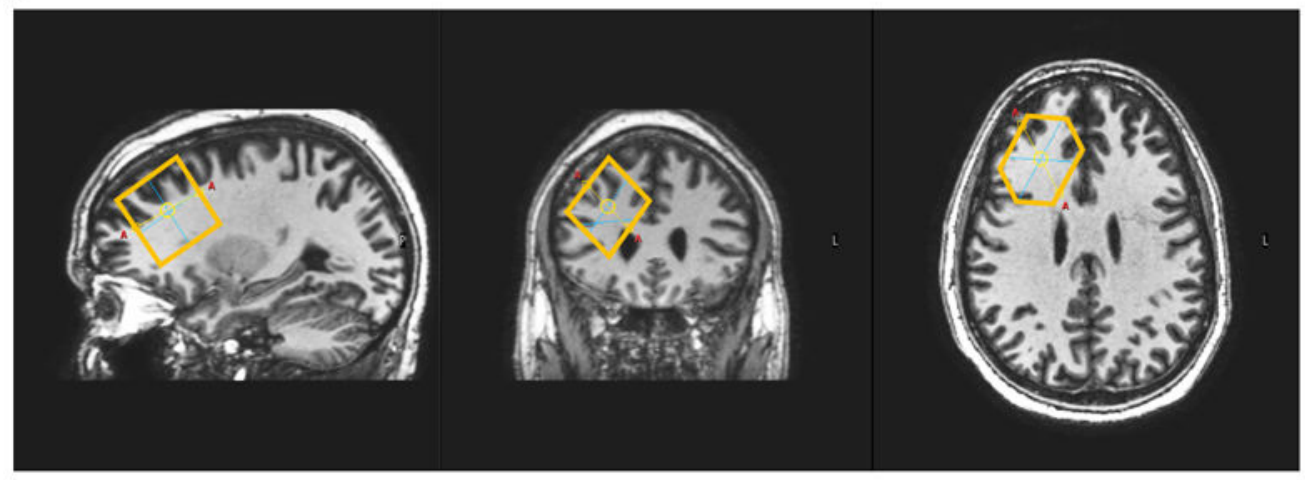

B

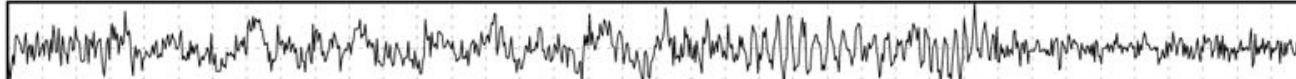
(NAA

Chemical Shift (ppm)

Figure 1. Voxel placement and sample spectra from dorsolateral prefrontal cortex (DLPFC)

A. From left to right: sagittal view, coronal view, and axial view of the voxel.

B. A typical fitted spectrum (in red). Indicated are myo-inositol (mI), choline (Cho), creatine (Cr), Glx and total NAA. Above the spectrum the residual signal after fitting is displayed. The baseline is displayed below the spectrum. 


\section{A}

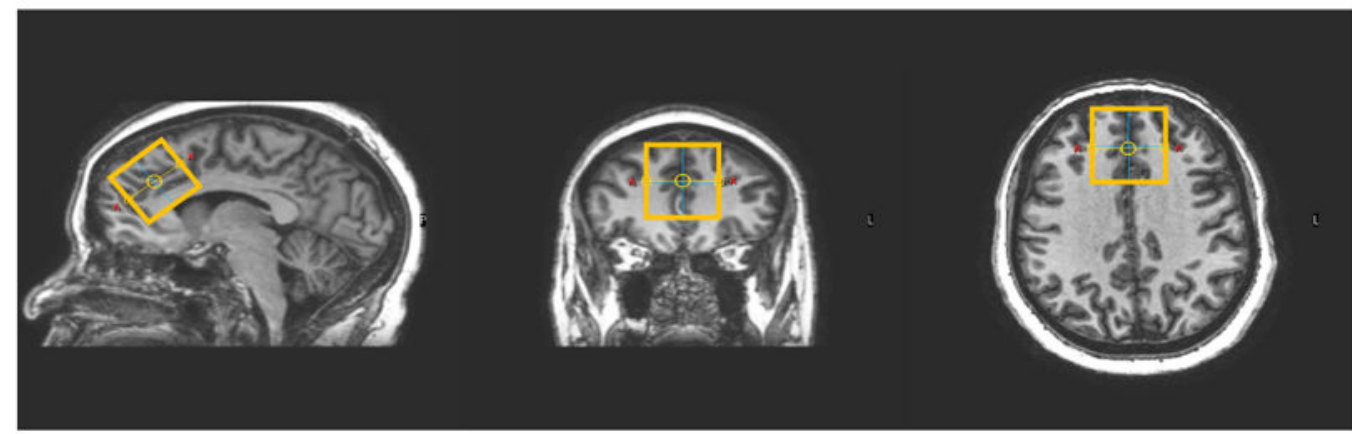

B

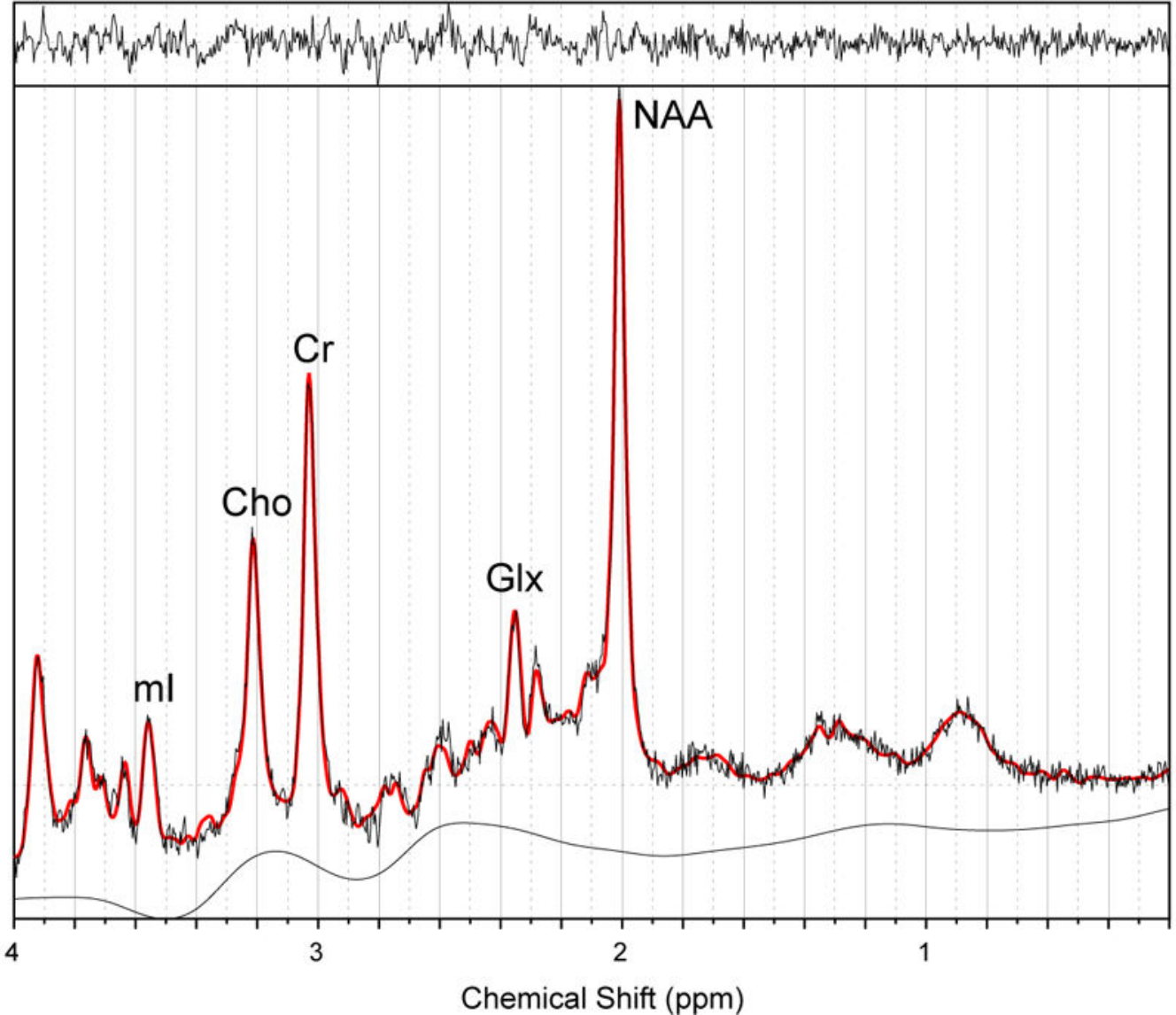

Figure 2. Voxel placement and sample spectra from anterior cingulate cortex (ACC)

A. From left to right: sagittal view, coronal view, and axial view of the voxel.

B. A typical fitted spectrum (in red). Indicated are myo-inositol (mI), choline (Cho), creatine $(\mathrm{Cr})$, Glx and total NAA. Above the spectrum the residual signal after fitting is displayed. The baseline is displayed below the spectrum. 
ACC

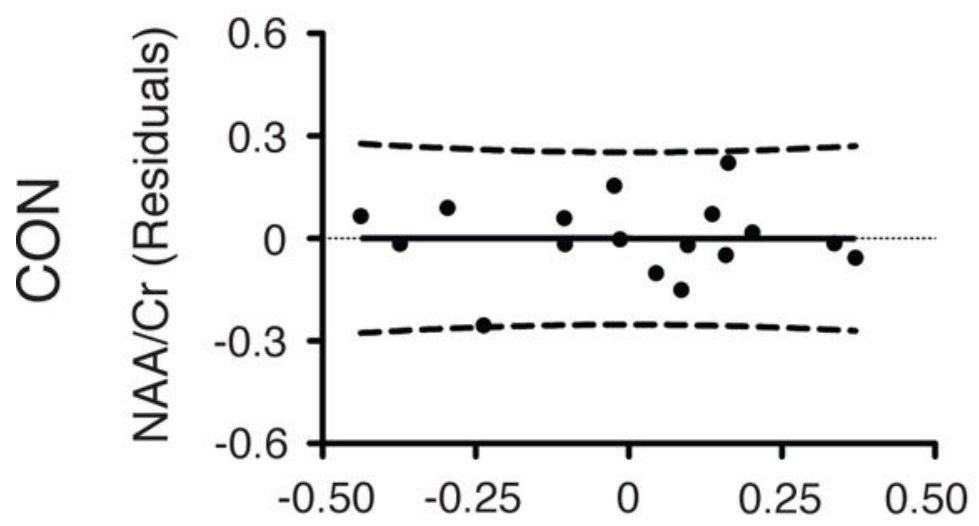

N

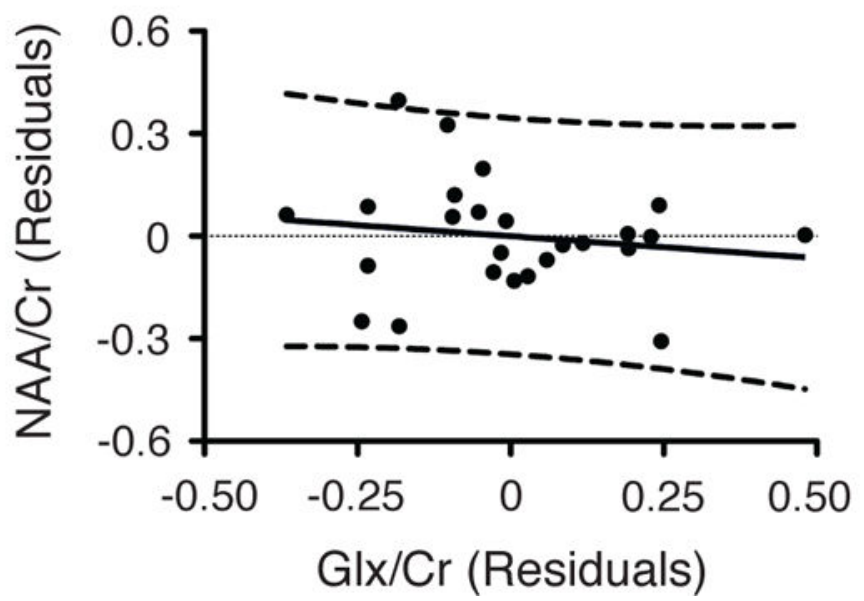

DLPFC
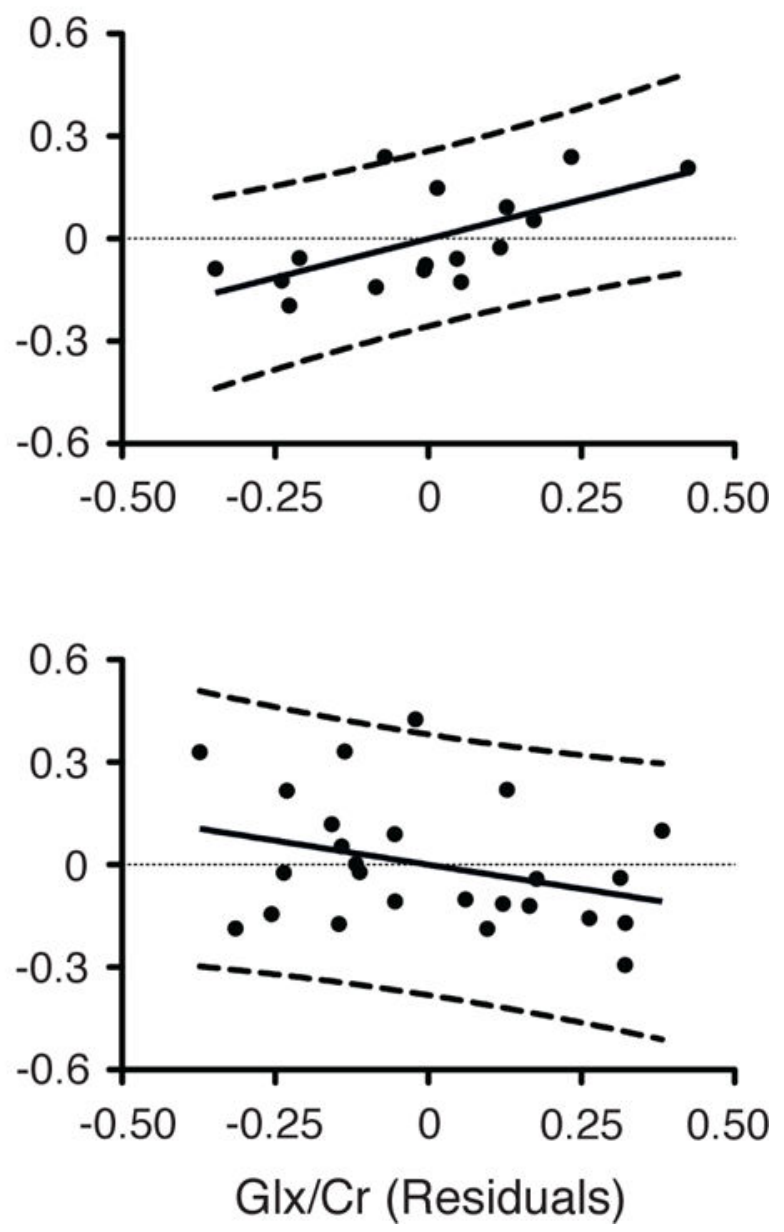

Figure 3. Correlation between $\mathrm{NAA} / \mathrm{Cr}$ and $\mathrm{Glx} / \mathrm{Cr}$

Adjusted partial correlations between NAA/Cr and Glx/Cr of healthy controls (upper row) and patients with schizophrenia (lower row) in ACC (left column) and DLPFC (right column). Partial correlations controlling for age and smoking status in ACC (HC: $\mathrm{r}=-0.002$, $P=0.996 ; \mathrm{SZ}: \mathrm{r}=-0.154, P=0.483)$ and in DLPFC $(\mathrm{HC}: \mathrm{r}=0.627, P=0.017$; SZ: $\mathrm{r}=$ $-0.330, P=0.124)$. 


\section{Table 1}

Clinical and Demographic Characteristics.

\begin{tabular}{|c|c|c|c|c|}
\hline & $\mathrm{HC}^{a}$ & SZ & $t$ test & $P$ value $^{b}$ \\
\hline $\begin{array}{l}\text { Age (years) } \\
\qquad(\mathrm{HC} / \mathrm{SZ}: 17 / 25)^{c}\end{array}$ & $32.29(9.96)$ & $33.84(11.53)$ & 0.450 & 0.655 \\
\hline $\begin{array}{l}\text { Gender (\% Male) } \\
\quad \text { (HC/SZ: } 17 / 25)\end{array}$ & 82.35 & 76.00 & & 0.716 \\
\hline $\begin{array}{l}\text { Years of Education } \\
\text { (HC/SZ: } 17 / 25)\end{array}$ & $13.12(1.83)$ & $13.00(2.18)$ & 0.183 & 0.856 \\
\hline $\begin{array}{l}\text { Smoking Status (\% Current Smoker) } \\
\text { (HC/SZ: } 17 / 25)\end{array}$ & 23.53 & 52.00 & & 0.109 \\
\hline $\begin{array}{l}\text { Ethnicity (\% African American, \% Caucasian, \% Asian, \% Mixed Ethnicity) } \\
\text { (HC/SZ: 17/25) }\end{array}$ & $82.4 / 17.6 / 0 / 0$ & $56.0 / 36.0 / 4 / 4$ & & 0.284 \\
\hline $\begin{array}{l}\text { SAPS } \\
(\text { HC/SZ: } 17 / 25)\end{array}$ & . & $12.16(21.59)$ & & \\
\hline $\begin{array}{l}\text { SANS } \\
\quad(\mathrm{HC} / \mathrm{SZ}: 17 / 25)\end{array}$ & . & $31.96(23.68)$ & & \\
\hline \multicolumn{5}{|l|}{ Neurocognitive Domains ${ }^{d}$} \\
\hline $\begin{array}{l}\text { Processing Speed } \\
\text { (HC/SZ: } 15 / 22)\end{array}$ & $100.20(12.73)$ & $86.45(16.29)$ & 2.743 & 0.010 \\
\hline $\begin{array}{l}\text { Attention } \\
\quad(\mathrm{HC} / \mathrm{SZ}: 14 / 20)\end{array}$ & $92.07(14.22)$ & $83.35(17.05)$ & 1.568 & 0.127 \\
\hline $\begin{array}{l}\text { Verbal Learning and Memory } \\
\text { (HC/SZ: } 15 / 23)\end{array}$ & 94.07 (16.77) & $83.43(19.11)$ & 1.757 & 0.087 \\
\hline $\begin{array}{l}\text { Visuospatial Memory } \\
\quad \text { (HC/SZ: } 15 / 23)\end{array}$ & $87.00(21.06)$ & $82.87(18.80)$ & 0.632 & 0.532 \\
\hline $\begin{array}{l}\text { Ideational Fluency } \\
\quad(\mathrm{HC} / \mathrm{SZ}: 15 / 23)\end{array}$ & $102.20(9.70)$ & $91.22(13.60)$ & 2.705 & 0.010 \\
\hline $\begin{array}{l}\text { Executive Function } \\
\quad(\mathrm{HC} / \mathrm{SZ}: 15 / 23)\end{array}$ & $93.73(16.29)$ & $98.26(17.01)$ & 0.815 & 0.420 \\
\hline
\end{tabular}

${ }^{a}$ Healthy Control (HC); Schizophrenia (SZ); Data in table is presented as Mean (Standard Deviation) unless otherwise indicated.

${ }^{b} P$ values for comparisons of continuous data (t test) and categorical data (Fisher's exact test).

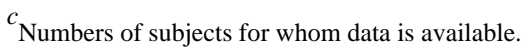

Domain scores presented for those patients and healthy controls who completed neurocognitive testing since three patients and three controls refused participation in this battery. 


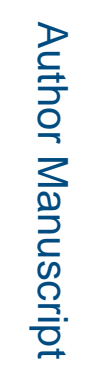

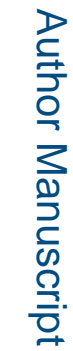

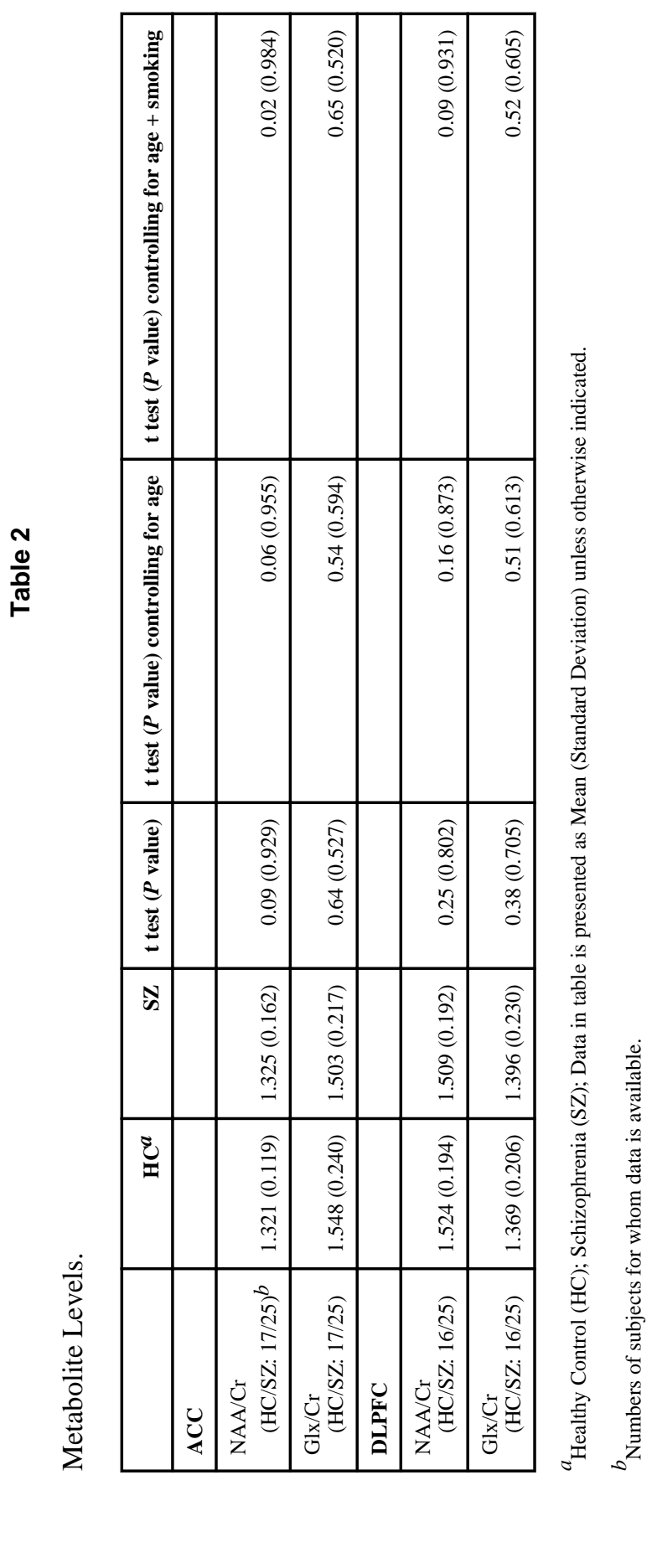

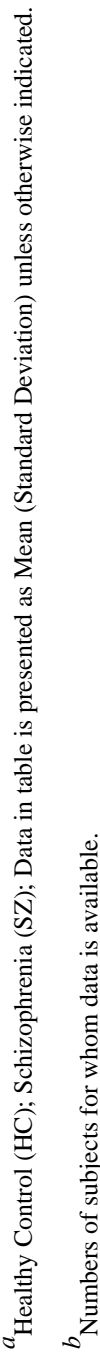

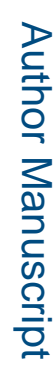

Curr Mol Med. Author manuscript; available in PMC 2015 July 21. 